\title{
WAVE PROPAGATION IN A MICROPOLAR TRANSVERSELY ISOTROPIC GENERALIZED THERMOELASTIC HALF-SPACE
}

\author{
R.R. GUPTA \\ Department of Mathematics and Applied Sciences \\ MEC, Muscat, OMAN \\ E-mail:dr.rajanigupta@gmail.com
}

\begin{abstract}
Rayleigh waves in a half-space exhibiting microplar transversely isotropic generalized thermoelastic properties based on the Lord-Shulman (L-S), Green and Lindsay (G-L) and Coupled thermoelasticty (C-T) theories are discussed. The phase velocity and attenuation coefficient in the previous three different theories have been obtained. A comparison is carried out of the phase velocity, attenuation coefficient and specific loss as calculated from the different theories of generalized thermoelasticity along with the comparison of anisotropy. The amplitudes of displacements, microrotation, stresses and temperature distribution were also obtained. The results obtained and the conclusions drawn are discussed numerically and illustrated graphically. Relevant results of previous investigations are deduced as special cases.
\end{abstract}

Key words: micropolar, transversely isotropic, generalized thermoelastic, amplitude ratios.

\section{Introduction}

Classical mechanics deals with the basic assumption that the effect of the microstructure of a material is not essential for describing its mechanical behavior. Such an approximation has been shown in many well-known cases. Often, however, discrepancies between the classical theory and experiments are observed, indicating that the microstructure might be important. For example, discrepancies have been found in the stress concentrations in the areas of holes, notches and cracks; elastic vibrations characterized by a high frequency and small wavelengths, particularly in granular composites consisting of stiff inclusions embedded in a weaker matrix, fibers or grains; and the mechanical behavior of complex fluids such as liquid crystals, polymeric suspensions, and animal blood. In general, granular composites, for example porous materials, are widely used in the area of passive noise control as sound absorbers and the effect of acoustical waves characterized by high frequencies and small wavelengths become significant.

To explain the fundamental departure of microcontinuum theories from the classical continuum theories, a continuum model with microstructures to describe the microscopic motion or a non local model to describe the long range material interaction is developed. This theory extends the application of the continuum model to microscopic space and short-time scales. The micromorphic theory (Suhubi and Eringen, 1964; Eringen, 1999) treats a material body as a continuous collection of a large number of deformable particles, with each particle possessing a finite size and inner structure. Using assumptions such as infinitesimal deformation and slow motion, the micromorphic theory can be reduced to Mindlin's microstructure theory (1964). When the microstructure of the material is considered rigid, it becomes the micropolar theory (Eringen, 1966).

Eringen's micropolar theory is more appropriate for geological materials like rocks, soils since, this theory takes into account the intrinsic rotation and predicts the behavior of a material with inner structure. Different researchers discussed different type of problems in a transversely isotropic elastic material. Abubakar (1962) discussed free vibrations of a transversely isotropic plate. Keck et al. (1971) derived the frequency equation for the propagation of train of a nontorsional axisymmetric harmonic wave in infinitely long shells, made of three concentric cylinders of different transversely isotropic materials. Shuvalov et al. 
(1974) described the long wavelength onset of the fundamental branches for a free anisotropic plate with an arbitrary through plate variation of material properties. Payton in 1991 studied wave propagation in a restricted transversely isotropic elastic solid whose surface contains conical points. However, no attempt has been made to study the wave propagation in a micropolar transversely isotropic medium.

The aim of the present study is to improve our knowledge about the propagation of waves in a micropolar transversely isotopic layer. This study has many applications in various fields of science and technology, namely, atomic physics, industrial engineering, thermal power plants, submarine structures, pressure vessel, aerospace, chemical pipes and metallurgy. After developing the solution, frequency equations connecting the phase velocity with the wave number, for symmetric and skew-symmetric wave modes are derived. The amplitude ratios of displacements and microrotation are also obtained. The dispersion curves, attenuation coefficients, amplitude ratio of displacements and microrotation for symmetric and skew-symmetric waves are presented and illustrated graphically to evince the effect of anisotropy.

\section{Basic equations}

Following Eringen (1999), the constitutive relations and balance laws in a general micropolar anisotropic medium possessing the center of symmetry, in the absence of body forces, body couples, are given by

\section{Balance laws}

$$
\begin{aligned}
& t_{j i, j}=\rho \ddot{u}_{i}, \\
& m_{i k, i}+\varepsilon_{i m n} t_{m n}=\rho j \ddot{\varphi}_{k} .
\end{aligned}
$$

\section{Constitutive relations}

$$
\begin{aligned}
& t_{i j}=A_{i j k l} E_{k l}+G_{i j k l} \Psi_{k l}-\beta_{i j}\left(1+\tau_{l} \frac{\partial}{\partial t}\right) T, \\
& m_{i j}=G_{j i k l} E_{k l}+B_{k l j i} \Psi_{k l} .
\end{aligned}
$$

\section{Heat conduction equation}

$$
K_{i j}^{*} T_{, j}=\rho c^{*}\left(1+\tau_{0} \frac{\partial}{\partial t}\right) \dot{T}+T_{0}\left(\frac{\partial}{\partial t}+n_{0} \tau_{0} \frac{\partial^{2}}{\partial t^{2}}\right) \beta_{i j} u_{i, j} .
$$

The deformation and wryness tensor are defined by

$$
E_{j i}=u_{i, j}+\varepsilon_{i j k} \varphi_{k}, \quad \Psi_{i j}=\varphi_{i, j} .
$$

The list of symbols is given in the nomenclature.

\section{Problem formulation and solution}

We have used appropriate transformations following Slaughter (2002), on the set of Eq.(2.1) to derive equations for a micropolar generalized thermoelastic transversely isotopic medium and restricted our analysis to the two dimensional problem. 
We consider a homogeneous, micropolar generalized thermoelastic transversely isotropic half-space initially in an undeformed state and at uniform temperature $T_{0}$. We take the origin of the coordinate system on the top plane surface and the $x_{3}$ axis pointing normally into the half-space, which is thus represented by $x_{3} \geq 0$. We choose the $x_{1}$-axis along the direction of wave propagation so that all particles on a line parallel to the $x_{2}$-axis are evenly displaced. Therefore, all the field quantities will be independent of the $x_{2}$ coordinate. Further, the disturbance is assumed to be confined to the neighborhood of the free surface and hence vanishes as $x_{3} \rightarrow \infty$. So, we assume the components of the displacement and microrotation vector of the form

$$
\boldsymbol{u}=\left(u_{1}, 0, u_{3}\right), \quad \boldsymbol{\varphi}=\left(0, \varphi_{2}, 0\right)
$$

Thus, the field equations reduce to

$$
\begin{aligned}
& A_{11} \frac{\partial^{2} u_{1}}{\partial x_{1}^{2}}+A_{55} \frac{\partial^{2} u_{1}}{\partial x_{3}^{2}}+\left(A_{13}+A_{56}\right) \frac{\partial^{2} u_{3}}{\partial x_{1} \partial x_{3}}+K_{1} \frac{\partial \varphi_{2}}{\partial x_{3}}-\beta_{1}\left(1+\tau_{1} \frac{\partial}{\partial t}\right) \frac{\partial T}{\partial x_{1}}=\rho \frac{\partial^{2} u_{1}}{\partial t^{2}} \\
& A_{66} \frac{\partial^{2} u_{3}}{\partial x_{1}^{2}}+A_{33} \frac{\partial^{2} u_{3}}{\partial x_{3}^{2}}+\left(A_{13}+A_{56}\right) \frac{\partial^{2} u_{1}}{\partial x_{1} \partial x_{3}}+K_{2} \frac{\partial \varphi_{2}}{\partial x_{1}}-\beta_{3}\left(1+\tau_{1} \frac{\partial}{\partial t}\right) \frac{\partial T}{\partial x_{3}}=\rho \frac{\partial^{2} u_{3}}{\partial t^{2}} \\
& B_{77} \frac{\partial^{2} \varphi_{2}}{\partial x_{1}^{2}}+B_{66} \frac{\partial^{2} \varphi_{2}}{\partial x_{3}^{2}}-K_{1} \frac{\partial u_{1}}{\partial x_{3}}+K_{1} \frac{\partial \varphi_{2}}{\partial x_{3}}=\rho \frac{\partial^{2} u_{1}}{\partial t^{2}}, \\
& K_{1}^{*} \frac{\partial^{2} T}{\partial x_{1}^{2}}+K_{3}^{*} \frac{\partial^{2} T}{\partial x_{3}^{2}}=\rho c^{*}\left(1+\tau_{0} \frac{\partial}{\partial t}\right) \frac{\partial T}{\partial t}+T_{0}\left(1+n_{0} \tau_{0} \frac{\partial}{\partial t}\right)\left(\beta_{1} \frac{\partial \dot{u}_{1}}{\partial x_{1}}+\beta_{3} \frac{\partial \dot{u}_{3}}{\partial x_{3}}\right) \\
& t_{33}=A_{11} \frac{\partial u_{1}}{\partial x_{1}}+A_{33} \frac{\partial u_{3}}{\partial x_{3}}-\beta_{3}\left(1+\tau_{1} \frac{\partial}{\partial t}\right) T, \\
& t_{31}=A_{65} \frac{\partial u_{3}}{\partial x_{1}}-K_{1} \varphi_{2}+A_{55} \frac{\partial u_{1}}{\partial x_{3}}, \quad m_{32}=B_{66} \frac{\partial \varphi_{2}}{\partial x_{3}}
\end{aligned}
$$

where

$$
K_{1}=A_{56}-A_{55}, \quad K_{2}=A_{66}-A_{56}, \quad X=K_{2}-K_{1},
$$

and we have used the notations $11 \rightarrow 1,33 \rightarrow 3,12 \rightarrow 7,13 \rightarrow 6,23 \rightarrow 5$ for the material constants.

For the Lord and Shulman (L-S) theory we take $\tau_{1}=0, n_{0}=1$, for Green and Lindsay (G-L) theory we take $\tau_{1} \geq \tau_{0} \geq 0, n_{0}=0$, and for the coupled theory (CT) $\tau_{1}=\tau_{0}=0, n_{0}=0$.

For further considerations, it is convenient to introduce the dimensionless variables defined by 


$$
\begin{aligned}
& \left(x_{1}^{\prime}, x^{\prime}\right)_{3}=\frac{\omega^{*}}{c_{1}}\left(x_{1}, x_{3}\right), \quad\left(u_{1}^{\prime}, u^{\prime}\right)_{3}=\frac{\omega^{*}}{c_{1}}\left(u_{1}, u_{3}\right), \quad t_{i j}^{\prime}=\frac{t_{i j}}{A_{11}}, \\
& m_{i j}^{\prime}=\frac{m_{i j} c_{1}}{B_{56} \omega^{*}}, \quad \varphi_{2}^{\prime}=\frac{\varphi_{2} A_{55}}{K_{1}}, \quad T^{\prime}=\frac{T}{T_{0}}, \\
& t^{\prime}=\omega^{*} t, \quad \tau_{0}^{\prime}=\omega^{*} \tau_{0}, \quad \tau_{1}^{\prime}=\omega^{*} \tau_{1}, \quad \omega^{* 2}=\frac{X}{\rho j}, \quad c_{1}^{2}=\frac{A_{11}}{\rho} .
\end{aligned}
$$

\section{Normal mode analysis and solution of the problem}

We assume the solution for representing propagating waves in the plane of the form

$$
\left(u_{1}, u_{3}, \varphi_{2}, T\right)=\left(1, \bar{u}_{3}, \bar{\varphi}_{2}, \bar{T}\right) u_{1} e^{i \xi\left(x_{1}+m x_{3}-c t\right)}
$$

where $\xi$ is the wave number, $\omega=\xi_{c}$ is the angular frequency and $c$ is the phase velocity of the wave, $m$ is the unknown parameter which signifies the penetration depth of the wave, $\bar{u}_{3}, \bar{\varphi}_{2}, \bar{T}$ are respectively, the amplitude ratios of the displacement $u_{3}$, microrotation $\varphi_{2}$ and temperature distribution to that of the displacement $u_{1}$.

With the help of Eqs (3.7) and (3.8), field Eqs (3.2)-(3.5) are reduced to (after suppressing primes)

$$
\begin{aligned}
& {\left[-m^{2} \xi^{2}+a_{1}-d_{2} m \xi^{2} \bar{u}_{3}+i \xi m d_{3} \bar{\varphi}_{2}+a_{2} \bar{T}\right] u_{1} e^{i \xi\left(x_{1}+m x_{3}-c t\right)}=0} \\
& {\left[-m \xi^{2} d_{5}+\left(-m^{2} \xi^{2}+a_{3}\right) \bar{u}_{3}+i \xi d_{6} \bar{\varphi}_{2}+m a_{4} \bar{T}\right] u_{1} e^{i \xi\left(x_{1}+m x_{3}-c t\right)}=0} \\
& {\left[-i \xi m d_{9}-i \xi m d_{10} \bar{u}_{3}+\left(-m^{2} \xi^{2}+a_{5}\right) \bar{\varphi}_{2}\right] u_{1} e^{i \xi\left(x_{1}+m x_{3}-c t\right)}=0,} \\
& a_{6}+a_{7} \bar{u}_{3}+\left(m^{2} \xi^{2}+a_{8}\right) \bar{T}=0
\end{aligned}
$$

where

$$
\begin{aligned}
& a_{1}=-d_{1} \xi^{2}+\omega^{2}, \quad a_{2}=-i \xi d_{13}\left(1-i \omega \tau_{1}\right), \quad a_{3}=-d_{4} \xi^{2}+\omega^{2} d_{7}, \\
& a_{4}=-i \xi d_{14}\left(1-i \omega \tau_{1}\right), \quad a_{5}=-d_{8} \xi^{2}-d_{11}+\omega^{2} d_{12}, \quad a_{6}=\varepsilon_{2} \bar{\beta} \omega \xi\left(1-i \omega n_{0} \tau_{0}\right), \quad a_{7}=a_{6} / \bar{\beta}, \\
& d_{1}=A_{11} / A_{55}, \quad d_{2}=\left(A_{13} /+A_{56}\right) / A_{55}=d_{5}, \quad d_{3}=K_{1}^{2} / A_{55}^{2}, \\
& d_{4}=A_{66} / A_{33}, \quad d_{6}=K_{1} K_{2} / A_{33} A_{55}, \quad d_{7}=A_{55} / A_{33}, \quad d_{8}=B_{77} / B_{66}, \\
& d_{9}=-A_{55} c_{1}^{2} / B_{66} \omega^{* 2}, \quad d_{10}=-K_{2} A_{55} c_{1}^{2} / K_{1} B_{66} \omega^{* 2}, \quad d_{11}=-X c_{1}^{2} / B_{66} \omega^{* 2},
\end{aligned}
$$




$$
\begin{aligned}
& d_{12}=A_{55} j / B_{66}, \quad d_{13}=\beta_{1} T_{0} / A_{33}, \quad d_{14}=\beta_{3} T_{0} / A_{33}, \quad \varepsilon_{1}=\rho c^{*} c_{1}^{2} / K_{3} \omega^{*}, \\
& \varepsilon_{2}=\beta_{3} c_{1}^{2} / K_{3} \omega^{*}, \quad \bar{K}=K_{1} / K_{3}, \quad \bar{\beta}=\beta_{1} / \beta_{3}, \quad d_{15}=K_{1}^{2} / A_{55}^{2}, \quad d_{16}=A_{65} / A_{55} .
\end{aligned}
$$

The condition for the non trivial solution of the system of Eq.(4.2), yields a biquadratic equation in $q\left(=m^{2}\right)$ as

$$
A q^{4}+B q^{3}+C q^{2}+D q+E=0
$$

where

$$
\begin{aligned}
& A=-\xi^{8}, \quad B=-\xi^{4} a_{4} a_{7}+\xi^{6}\left(a_{3}+a_{5}+a_{1}-a_{8}+d_{3} d_{9}\right)+\xi^{8} d_{2} d_{5}, \\
& C=\xi^{4}\left[a_{2}\left(d_{5} a_{7}-a_{6}\right)-a_{3} a_{5}+a_{4} d_{2} a_{6}+d_{6} d_{10}+\right. \\
& \left.-\left(a_{1}-a_{8}\right)\left(a_{3}+a_{5}\right)+a_{1} a_{8}-d_{3} d_{9}\left(a_{7}-a_{8}\right)\right]+ \\
& +\xi^{2} a_{7}\left(a_{4} a_{1}+a_{5}-d_{3} d_{9}\right)-\xi^{6}\left(d_{2} d_{5} a_{5}+d_{2} d_{6} d_{9}+d_{3} d_{5} d_{10}\right), \\
& D=\xi^{2}\left[a_{2} a_{3} a_{6}-a_{2} a_{5}\left(d_{5} a_{7}-a_{6}\right)+a_{7} d_{6} d_{9} a_{2}+\right. \\
& \left.-a_{4} a_{6}\left(a_{5} d_{2}+d_{3} d_{10}\right)+\left(a_{1}-a_{8}\right) a_{3} a_{5}-a_{1} a_{8}\left(a_{3}+a_{5}\right)-d_{3} d_{9} a_{7} a_{8}\right]+ \\
& -a_{4} a_{5} a_{1} a_{7}-\xi^{4}\left[\left(a_{1}-a_{8}\right) d_{6} d_{10}+d_{2}\left(d_{5} a_{5} a_{8}+d_{6} d_{9} d_{8}\right)+d_{3} d_{5} d_{10} d_{8}\right], \\
& E=a_{2}\left(\xi^{2} d_{6} a_{6} d_{10}-a_{5} a_{3} a_{6}\right)+a_{1} a_{8}\left(a_{3} a_{5}-\xi^{2} d_{6} d_{10}\right) .
\end{aligned}
$$

The complex coefficients in Eq.(4.3) implies that four roots, $q_{j},(j=1,2,3,4)$ of this equation may be complex. The complex phase velocities of the quasi-waves, will be varying with the direction of phase propagation. Therefore, the three waves propagating in such a medium are attenuating waves. These waves are called quasi-waves because polarisations may not be along the dynamic axis. Analogous to the propagation in a micropolar isotropic thermoelastic medium, these coupled waves may be called quasilongitudinal displacement (QLD) waves, quasi-transverse microrotational (QCTM) waves, quasi- transverse displacement (QCTD) waves and quasi-thermal waves (QCT) that are propagating with the descending phase velocities respectively.

\section{Boundary condition}

The surface $x_{3}=0$ of the half-space is assumed to satisfy the following boundary conditions

$$
t_{33}=0, \quad t_{31}=0, \quad m_{32}=0, \quad \frac{\partial T}{\partial x_{3}}+h T=0
$$

where $h \rightarrow 0$ is the surface heat transfer coefficient;

$h \rightarrow 0$ corresponds to thermally insulated boundaries and

$h \rightarrow \infty$ refers to isothermal boundaries. 


\section{Rayleigh wave equation}

To obtain the solution for surface waves, it is essential that motion is confined to the free surface $x_{3}=0$ of the half-space, so that the characteristic roots $\left(q_{j}\right)$ must satisfy the radiation conditions $\left(\operatorname{Re}\left(q_{j}\right) \geq 0\right)$. So, we take the solution for the displacement, microrotation and temperature distribution of the form

$$
\left(u_{1}, u_{3}, \varphi_{2}, T\right)=\sum_{j=1}^{4} A_{j}\left(1, r_{j}, s_{j}, t_{j}\right) e^{i \xi\left(x_{1}-c t+i x_{3} m_{j}\right)}
$$

where

$$
\begin{aligned}
& r_{j}=\frac{I I}{I}, \quad s_{j}=\frac{I I I}{I}, \quad t_{j}=\frac{I V}{I}, \\
& I=\left|\begin{array}{ccc}
-m_{j}^{2} \xi^{2}+a_{3} & i \xi d_{6} & m_{j} a_{4} \\
-i \xi d_{10} & -m_{j}^{2} \xi^{2}+a_{5} & 0 \\
m_{j} a_{7} & 0 & m_{j}^{2} \xi^{2}+a_{8}
\end{array}\right|, \quad I I=\left|\begin{array}{ccc}
-m_{j} \xi^{2} d_{5} & i \xi d_{6} & m_{j} a_{4} \\
-i m_{j} \xi d_{9} & -m_{j}^{2} \xi^{2}+a_{5} & 0 \\
a_{6} & 0 & m_{j}^{2} \xi^{2}+a_{8}
\end{array}\right|, \\
& I I I=\left|\begin{array}{ccc}
-m_{j} \xi^{2} d_{5} & -m_{j}^{2} \xi^{2}+a_{3} & m_{j} a_{4} \\
-i m_{j} \xi d_{9} & -i \xi d_{10} & 0 \\
a_{6} & m_{j} a_{7} & m_{j}^{2} \xi^{2}+a_{8}
\end{array}\right|, \quad I V=\left|\begin{array}{ccc}
-m_{j} \xi^{2} d_{5} & -m_{j}^{2} \xi^{2}+a_{3} & i \xi d_{6} \\
-i m_{j} \xi d_{9} & -i \xi d_{10} & -m_{j}^{2} \xi^{2}+a_{5} \\
a_{6} & m_{j} a_{7} & 0
\end{array}\right| .
\end{aligned}
$$

Using Eq.(5.2) in the boundary condition $(5,1)$, we obtain the frequency equation of the Rayleigh wave in micropolar generalized thermoelastic transversely isotropic half-space of the form

$$
m_{1} m_{2} A T 1+m_{1} m_{3} A T 2+m_{1} m_{4} A T 3+m_{2} m_{3} A T 4+m_{2} m_{4} A T 5+m_{3} m_{4} A T 6=0
$$

where

$$
\begin{array}{ll}
A T 1=\left(s_{1} t_{2}-s_{2} t_{1}\right)\left(a_{3}^{*} b_{4}^{*}-a_{4}^{*} b_{3}^{*}\right), & A T 2=\left(s_{1} t_{3}-s_{3} t_{1}\right)\left(a_{4}^{*} b_{2}^{*}-a_{2}^{*} b_{4}^{*}\right), \\
A T 3=\left(s_{1} t_{4}-s_{4} t_{1}\right)\left(a_{2}^{*} b_{3}^{*}-a_{3}^{*} b_{2}^{*}\right), & A T 4=\left(s_{2} t_{3}-s_{3} t_{2}\right)\left(a_{1}^{*} b_{4}^{*}-a_{4}^{*} b_{1}^{*}\right), \\
A T 5=\left(s_{2} t_{4}-s_{4} t_{2}\right)\left(a_{3}^{*} b_{1}^{*}-a_{1}^{*} b_{3}^{*}\right), & A T 6=\left(s_{3} t_{4}-s_{4} t_{3}\right)\left(a_{1}^{*} b_{2}^{*}-a_{2}^{*} b_{1}^{*}\right), \\
a_{j}^{*}=i \xi d_{1}-d_{7} r_{j} m_{j} \xi-d_{14} t_{j} / d_{7}, & b_{j}^{*}=i \xi d_{16} r_{j}-m_{j} \xi+s_{j} d_{15}^{2}, \quad j=1, . .4 .
\end{array}
$$




\section{Amplitudes of stresses and temperature distribution}

The amplitudes of stresses and temperature distribution for plane waves can be obtained as

$$
\begin{array}{ll}
t_{33}=\sum_{j=1}^{4} A_{i} a_{j}^{*} e^{i \xi\left(x_{1}-c t+i m_{i} x_{2}\right)}, & t_{31}=\sum_{j=1}^{4} A_{i} b_{j}^{*} e^{i \xi\left(x_{1}-c t+i m_{i} x_{2}\right)}, \\
m_{32}=\sum_{j=1}^{4} A_{i} s_{j} m_{j} e^{i \xi\left(x_{1}-c t+i m_{i} x_{2}\right)}, & t_{33}=\sum_{j=1}^{4} A_{i} a_{j}^{*} e^{i \xi\left(x_{1}-c t+i m_{i} x_{2}\right)} .
\end{array}
$$

\section{Particular case:}

(i) The resulting expressions of stresses and temperature distribution for coupled theory (C-T) can be obtained by substituting $\tau_{1}=\tau_{0}=0, n_{0}=0$ in Eq. $(5,4)$.

(ii) Taking, $\tau_{1}=0, n_{0}=1$ in Eq.(5.4), we obtain the expressions of stresses and temperature distribution corresponding to the Lord and Shulman (L-S) theory.

(iii) To obtain the expressions of stresses and temperature distribution corresponding to the Green and Lindsay (G-L) theory we substitute $\tau_{1} \geq \tau_{0} \geq 0, n_{0}=0$ in Eqs (5.4).

\section{Numerical results and discussion}

In order to illustrate the theoretical results obtained in the preceding sections, we now present some numerical results. For numerical computations, we take the values for relevant parameters for the micropolar transversely isotropic thermoelastic solid as

$$
\begin{array}{ll}
A_{11}=13.8 \times 10^{10} \mathrm{Nm}^{-2}, & A_{33}=14.43 \times 10^{10} \mathrm{Nm}^{-2}, \\
A_{55}=3.7 \times 10^{10} \mathrm{Nm}^{-2}, & A_{66}=4.2 \times 10^{10} \mathrm{Nm}^{-2}, \\
A_{13}=8.85 \times 10^{10} \mathrm{Nm}^{-2}, & A_{56}=2.977 \times 10^{10} \mathrm{Nm}^{-2}, \\
B_{77}=3.71 \times 10^{9} \mathrm{~N}, & B_{66}=3.9 \times 10^{9} \mathrm{~N}, \\
\rho=1.74 \mathrm{Kg} / \mathrm{m}^{3}, & j=0.2 \times 10^{-15} \mathrm{~m}^{2}, \\
K^{*}=0.6 \times 10^{-2} \mathrm{~J} / \mathrm{msec}^{o} \mathrm{C}, & C^{*}=0.23 \mathrm{~J} / \mathrm{Kg}^{o} \mathrm{C}, \quad T=298 \mathrm{~K} .
\end{array}
$$

Following Gauthier (1982) we take the non dimensional values for aluminium epoxy like composite 


$$
\begin{array}{ll}
\rho=2.19 \times 10^{3} \mathrm{Kgm}^{3}, & \lambda=9.4 \times 10^{10} \mathrm{Nm}^{-2}, \quad \mu=4 \times 10^{10} \mathrm{Nm}^{-2}, \quad K=1 \times 10^{10} \mathrm{Nm}^{-2}, \\
C^{*}=1.04 \mathrm{CalK}^{-1}, & \gamma=0.779 \times 10^{5} \mathrm{~N}, \quad j=0.2 \times 10^{-4} \mathrm{~m}^{-2} .
\end{array}
$$

All numerical computations are carried out for a single fixed value of frequency $\omega=.75$ and for two given values of the wave number $\xi=.25, .35$. The computations were carried out within the range $0 \leq x_{1} \leq 10$.

The solid lines represent the case of the micropolar thermoelastic transversely isotropic solid (MTIS) and the broken lines represent the case of the micropolar thermoelastic isotropic solid (MIS). A comparison of the three theories of generalized thermoelasticity, namely, coupled thermoelasticity (C-T), Lord Shulman (L-S) and Green Lindsay (G-L) is shown in all the graphs. The solid and dotted line without the center symbol corresponds to the $\mathrm{C}-\mathrm{T}$ theory, solid and dotted line with the center symbol $\left(-O-O_{-}\right)$corresponds to the L-S theory and the solid and dotted line with the center symbol $(-\times-\times-)$ corresponds to the case of the G-L theory.

Figures 1-2 show the variation of phase velocity and specific loss with the wave number and Figs 3-9 show the variation of stresses, displacements, microrotation and temperature distribution with respect to distance.

It follows from Fig. 1 that the variation of phase velocity for both MTIS and MIS decreases sharply and then attains a constant value with an increase in the wave number. Figure 2 presents that the value of specific loss initially oscillates within the interval $(0,2)$ and then becomes constant with the wave number for both MTIS and MIS. Figures 3 and 4 show that the value of normal stress and tangential stress increases sharply within the interval $(0,2)$ and then decreases to become constant at the end. The variations for all the 3 theories are similar with a slight difference in their amplitude. It follows from Fig.5 that the value of tangential couple stress initially goes on increasing with an increase in the distance up to a value 6 of distance, but after that it decreases with a further increase in the distance, when the wave number is .25 . However, as the value of the wave number gets increased, the value of tangential couple stress initially oscillates and then decreases.

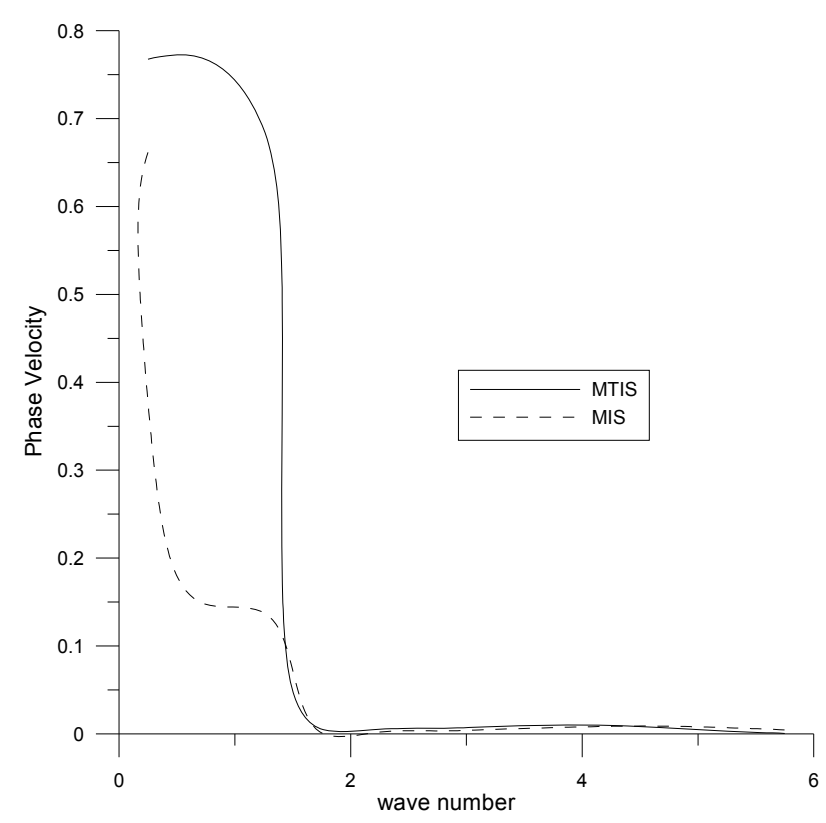

Fig.1. Variation of phase velocity with wave number.

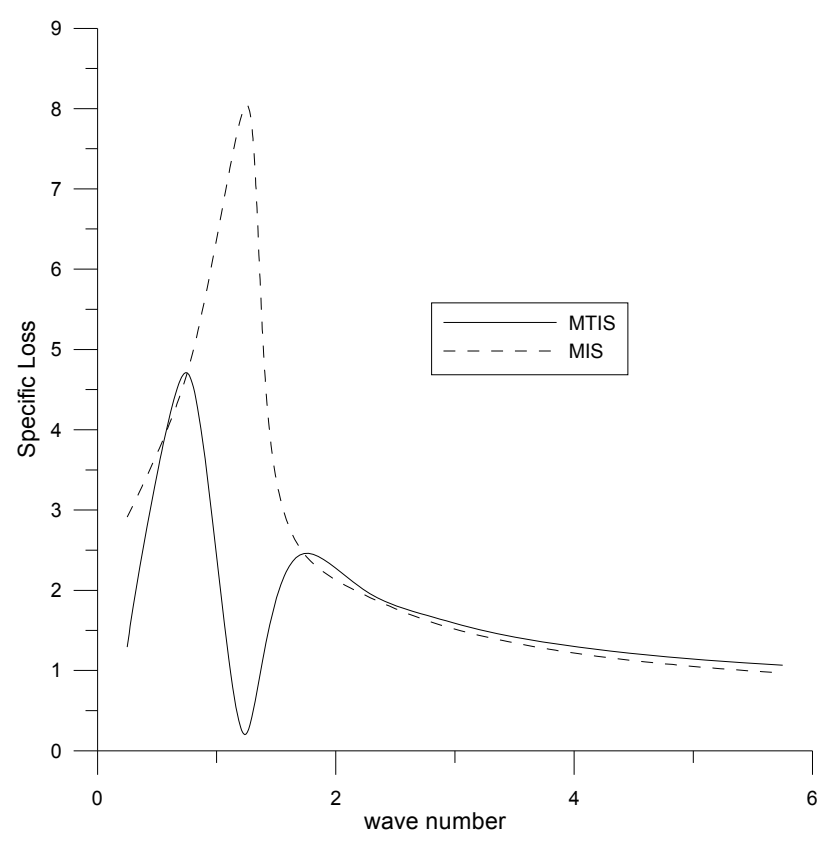

Fig.2. Variation of specific loss with wave number. 


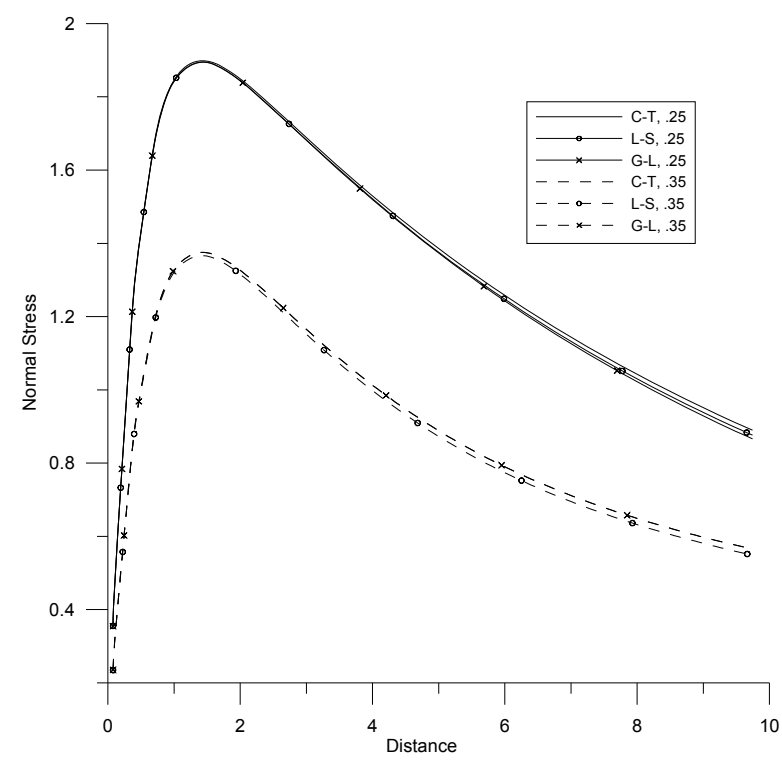

Fig.3. Variations of normal stress with distance.

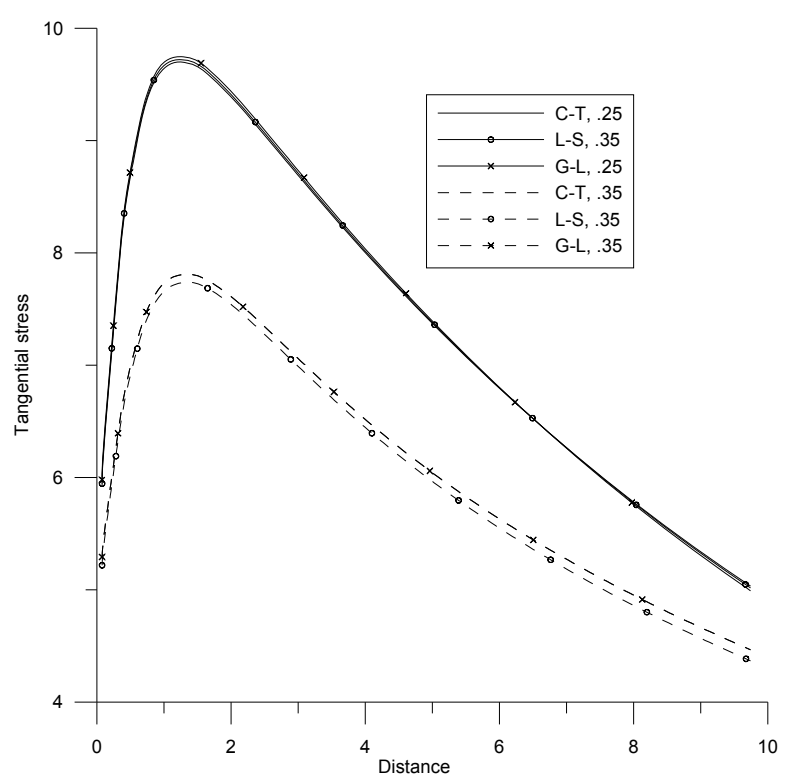

Fig.4. Variations of tangential stress with distance.

Figures 6-9 depict the variations of amplitudes and displacement, microrotation and temperature distribution with distance. It is illustrated in these figures that the value of displacements and microrotation go on decreasing as the distance from the surface increases. This variation pattern is physically admissible since the characteristics of Rayleigh waves are that the amplitude of the wave decreases rapidly with the depth. The rate of the decrease depends on the wavelength. Figure 9 shows that the value of temperature distribution initially increases at a depth of 5 units from the surface and then goes on decreasing with an increase in the distance. The value of displacements and microrotation gets decreased with an increase in the wave number. The variation pattern for all the three theories of thermoelasticity varies in the same manner with a slight difference in magnitude.

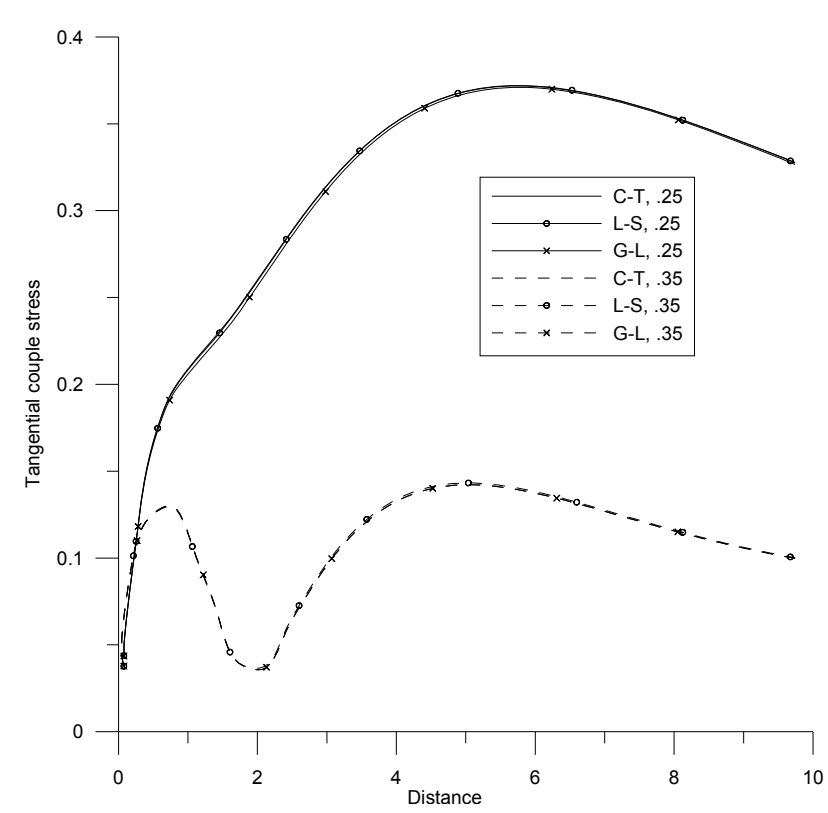

Fig.5. Variations of tangential couple stress with distance.

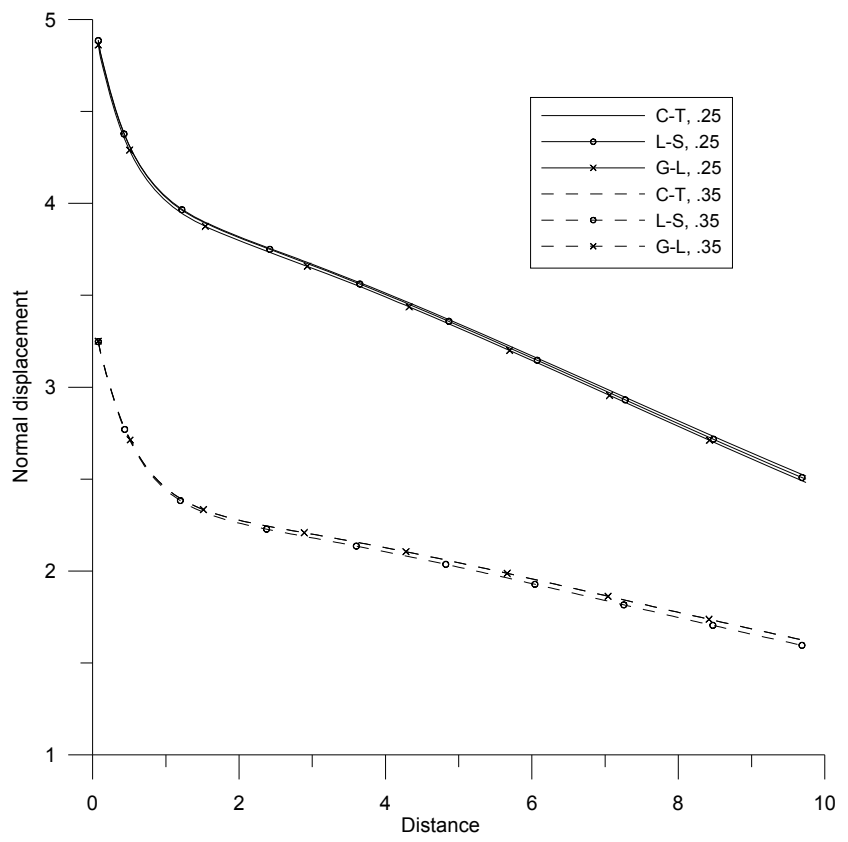

Fig.6. Variation of normal displacement with distance. 


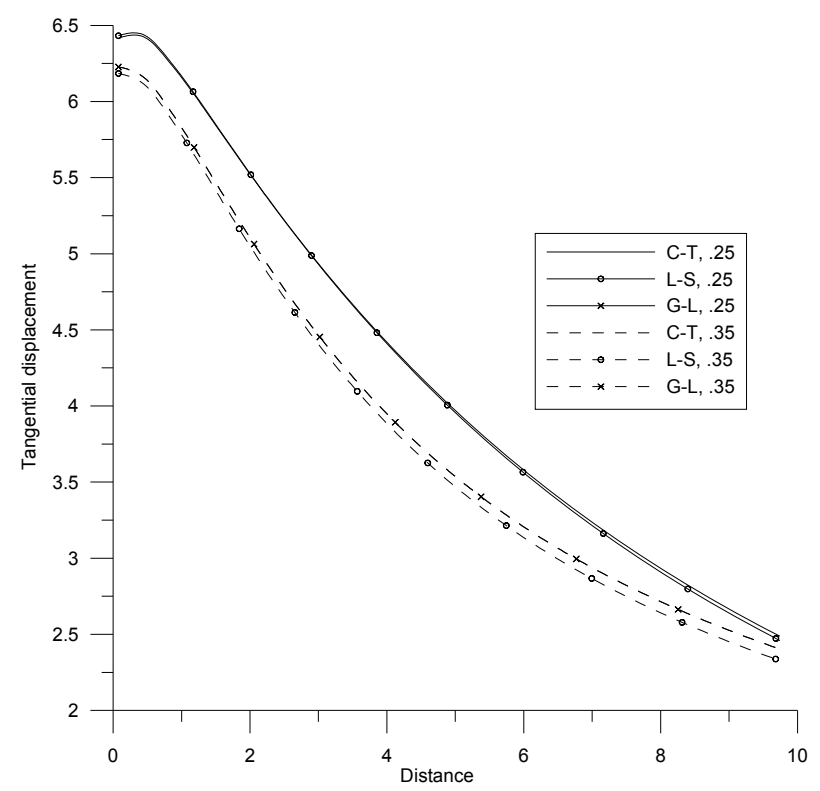

Fig.7. Variations of tangential displacement with distance.

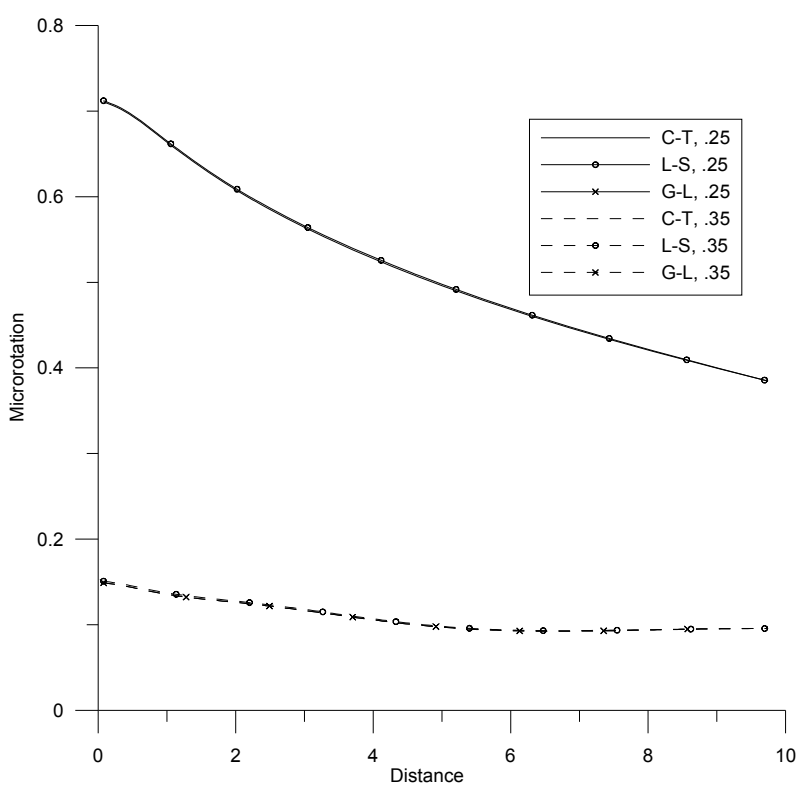

Fig.8. Variations of microrotation with distance.

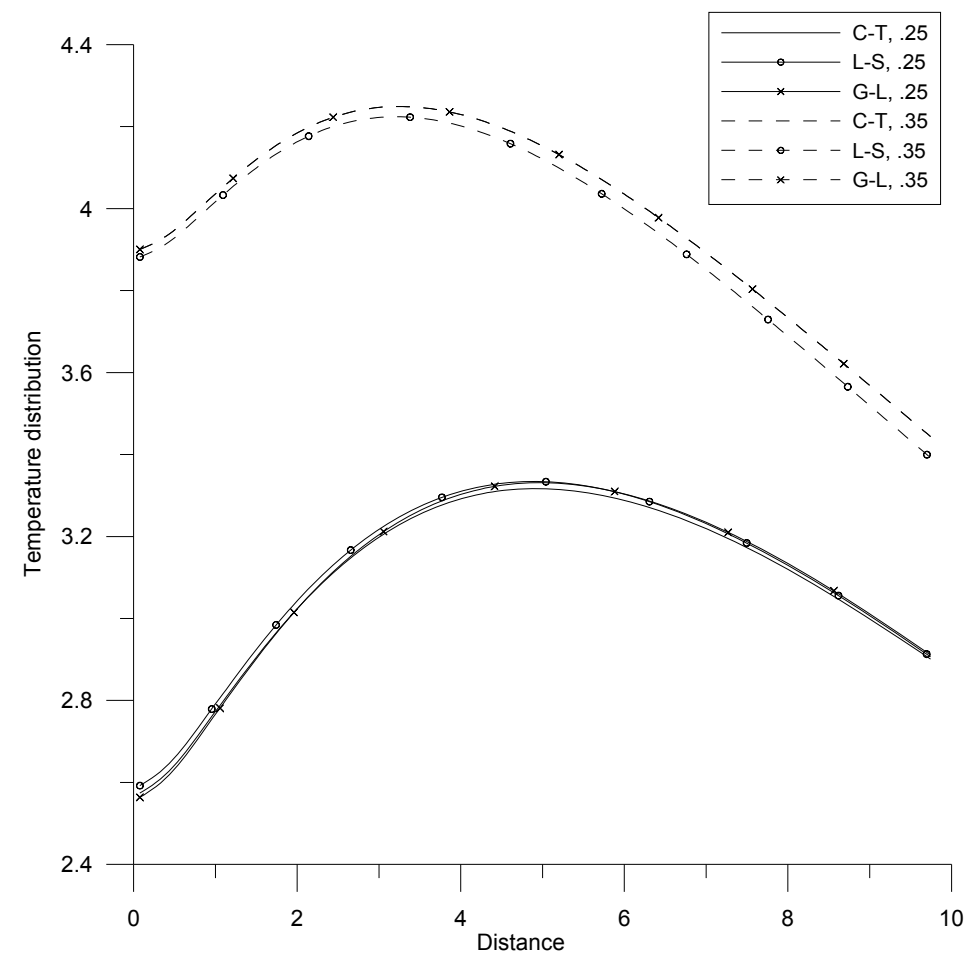

Fig.9. Variations of temperature distribution with distance.

\section{Conclusions}

The propagation of waves in a micropolar transversely isotropic medium possessing thermoelastic properties based on the Lord-Shulman (L-S), Green and Lindsay (G-L) and coupled thermoelasticty (C-T) theories are discussed. After developing the solution, the frequency equation for the propagation of Rayleigh waves has been derived. The expressions for amplitudes of stresses, displacements, microratation and 
temperature distribution have been derived and computed numerically. The numerical results have been plotted graphically. The results computed numerically are found to be in close agreement with the theoretical results.

\section{Nomenclature}

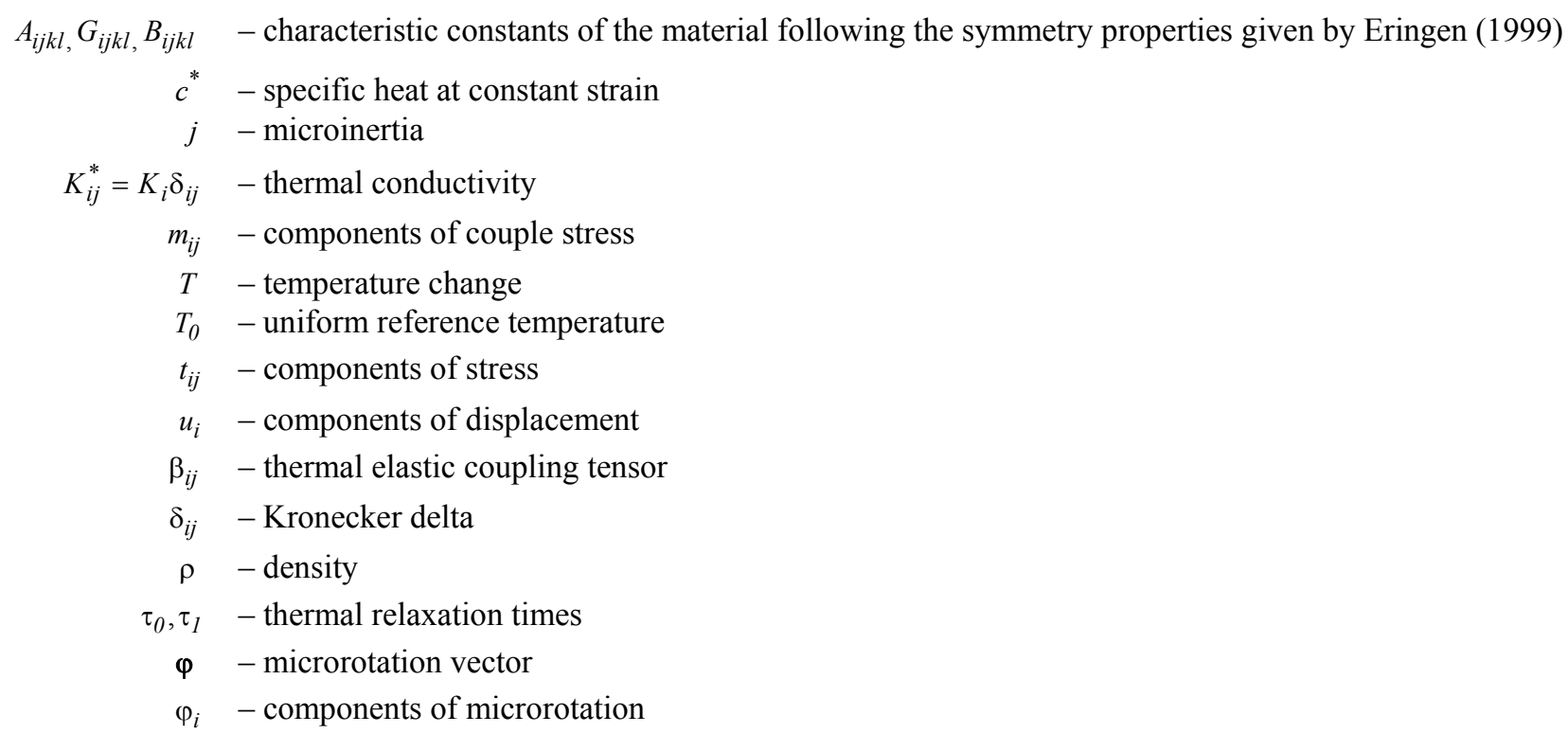

\section{References}

Abubakar I. (1962): Free vibrations of a transversely isotropic plate. - Quart. Journ. Mech. and Applied Math., vol.15, No.1, pp.129-136.

Eringen A.C. (1966): Linear theory of micropolar elasticity. - J. Math. Mech., vol.16, pp.909-923.

Eringen A.C. (1999): Microcontinuum Field Theories I: Foundations and Solids. - New York: Springer-Verlag.

Gauthier R.D. (1982). In experimental investigations on micropolar media. - Mechanics of Micropolar Media (eds) O. Brulin, RKT Hsieh. (Singapore: World Scientific)

Keck E. and Armenkas A.E. (1971): Wave propagation in transversely isotropic layered cylinders. - J. of the Engg. Mech. Division Procc. of the Amer. Soc. of Civil Engg., EM 2, pp.541- 555.

Mindlin R.D. (1964): Microstructure in linear elasticity. - Arch. Rational Mech. Anal., vol.16, pp.51-78.

Payton R.G. (1992): Wave propagation in a restricted transversely isotropic elastic solid whose slowness surface contains conical points. - Mech. Appl. Math., vol.45, Pt.2, pp.183-197.

Slaughter W.S. (2002): The Linearized Theory of Elasticity. - Birkhauser.

Suhubi E.S. and Eringen A.C. (1964): Non-linear theory of simple microelastic solids II. - Int. J. Engng. Sci., vol.2, pp.389-404.

Suvalov A.L., Poncelet O., Deschamps M. and Baron C. (2005): Long-wavelength dispersion of acoustic waves in transversely inhomogeneous anisotropic plates. - Wave Motion, vol.42, pp.367-382.

Received: May 22, 2013

Revised: August 29, 2013 